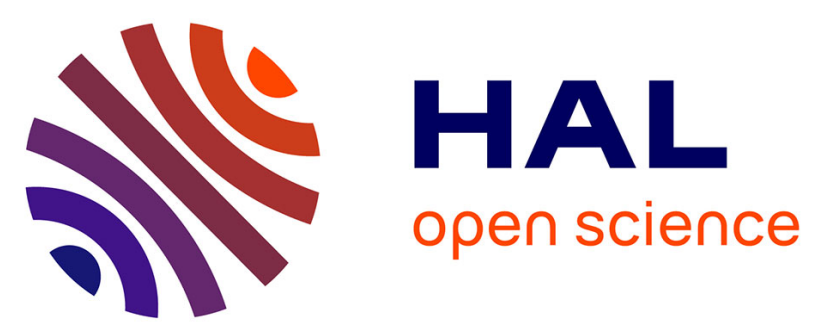

\title{
ILLUMINATION PROFILE CHARACTERIZATION OF A LIGHT DEVICE FOR THE DOSIMETRY OF INTRA-PLEURAL PHOTODYNAMIC THERAPY FOR MESOTHELIOMA
}

Camille Munck, Serge Mordon, Nacim Betrouni

\section{To cite this version:}

Camille Munck, Serge Mordon, Nacim Betrouni. ILLUMINATION PROFILE CHARACTERIZATION OF A LIGHT DEVICE FOR THE DOSIMETRY OF INTRA-PLEURAL PHOTODYNAMIC THERAPY FOR MESOTHELIOMA. Photodiagnosis and Photodynamic Therapy, 2016. hal-01366617

\section{HAL Id: hal-01366617 https://hal.science/hal-01366617}

Submitted on 15 Sep 2016

HAL is a multi-disciplinary open access archive for the deposit and dissemination of scientific research documents, whether they are published or not. The documents may come from teaching and research institutions in France or abroad, or from public or private research centers.
L'archive ouverte pluridisciplinaire HAL, est destinée au dépôt et à la diffusion de documents scientifiques de niveau recherche, publiés ou non, émanant des établissements d'enseignement et de recherche français ou étrangers, des laboratoires publics ou privés. 


\title{
Illumination profile characterization of a light device for the dosimetry of intra-pleural photodynamic therapy for mesothelioma
}

\author{
Camille Munck $^{1,2}$, Serge Mordon ${ }^{1}$, Nacim Betrouni $^{1}$
}

\begin{abstract}
${ }^{1}$ Univ. Lille, Inserm, CHU Lille, U1 189 - ONCO-THAI - Image Assisted Laser Therapy for Oncology, F-59000 Lille, France.

${ }^{2}$ Department of Pulmonary and Thoracic Oncology, Lille University Hospital, Lille, France
\end{abstract}

\author{
Corresponding Author \\ Nacim betrouni \\ INSERM U1189 Onco-Thai \\ 1, avenue Oscar Lambret \\ 59000, Lille, France. \\ Tel: 33320446722 \\ email : nacim.betrouni@inserm.fr
}




\section{ABSTRACT}

Background: Complete and homogeneous illumination of the pleural cavity is essential to the success of photodynamic therapy (PDT) for mesothelioma, but remains a challenge. Knowing the repartition and propagation of light around the light applicator could be the first step towards optimizing dosimetry. Here we propose a characterization method of the illumination profile of a specific light device.

Methods: The light wand, made of a cylindrical diffuser located inside an endotracheal tube, was fixed in a tank filled with dilute $0.01 \%$ intralipid. Light dosimetry was performed around the tip of the wand using two complementary methods: direct measurements of light power with an isotropic probe and measurements of light distribution characterization.

Results: Dosimetry with the isotropic probe showed an ellipse-shaped illumination. An optimized effective attenuation coefficient was deduced. Combined with the spatial representation, a theoretical illumination profile was established with iso-surfaces of fluence rate, defining a gradient light dose according to the distance from the diffuser.

Conclusion: A theoretical illumination profile of a light device was established and could be part of an intra-operative dosimetry system to improve light delivery during intrapleural PDT.

Keys words: Intrapleural photodynamic therapy, dosimetry, illumination profile, light device 


\section{INTRODUCTION}

Malignant pleural mesothelioma (MPM) is the primitive cancer of the pleura, occurring after exposure to asbestos fibers. It is a rare cancer, but its incidence is destined to rise throughout the world in the following decades, because of the increasing use of asbestos until the 1970's and since it is still used today in emerging countries. Its prognosis is poor with a median survival of less than a year, mainly due to complex treatments with disappointing outcomes. As part of a multimodal treatment, surgery offers the best chance at survival. However, even after the most complete tumor resection, microscopic tumor cells remain and it is essential to combine surgery with an efficient local adjuvant treatment. There are two main types of surgery: pleurectomy/decortication, where only the pleura is removed, and extra-pleural pneumonectomy, where the whole lung and the pleura are taken out. Whereas adjuvant hemithoracic radiotherapy or per operative intra-pleural therapies (chemotherapy, immunotherapy or genotherapy) have not proved to be satisfactory in terms of survival, intra-pleural photodynamic therapy (PDT) has recently become a valid candidate.

Friedberg et al. (Philadelphia, USA) have evaluated the impact of intra-pleural PDT after surgical resection of MPM $(1,2)$ and have demonstrated that adjuvant PDT improved the median survival significantly when associated with pleurectomy/decortication with a median survival was 31.7 months (95\% [CI] 9-54.3 months) (1). Their PDT protocol was the following (3): patients were administered a photosensitizer, Photofrin ${ }^{\circledR}$ (porfimer sodium), 48 hours before pleurectomy/decortications (P/D). Illumination of the pleural cavity was performed after maximal tumor resection, with a cylindrical light diffuser fixed inside an endotracheal tube and connected to a $635 \mathrm{~nm}$ laser source. To increase light diffusion, the pleural cavity and the cuff of the endotracheal tube were filled with a dilute intralipid solution of $0.01 \%$. The light dose to deliver was $60 \mathrm{~J} / \mathrm{cm}^{2}$. 
Illumination and dosimetry are key factors for a successful intra-pleural PDT, especially since the pleural cavity has a complex geometry. Knowing that, it seemed important to go back to the fundamentals of that light delivery and evaluate the light deployed by the device used for this procedure. The objective of this study was to produce an optimized light wand and propose a method to characterize the illumination profile of this light wand, in the clinical conditions of PDT for MPM. Characterization is done in term of fluence rate $\left(\mathrm{W} / \mathrm{cm}^{2}\right)$ measurements.

\section{MATERIAL AND METHODS}

\subsection{Light wand construction and setting}

Based on the device built by Friedberg et al. (3) a standardized light wand was designed using an endotracheal tube for structure. In addition, to ensure reproducibility for this study, the goals were to straighten the endotracheal tube for a better handling, and to fix the diffusing part of the optical fiber straight within the endotracheal tube and centered in the cuff. This light wand was composed of an endotracheal tube with an interior diameter of $8.5 \mathrm{~mm}$ (Super Safetyclear, Ruschelit ${ }^{\circledR}$, Teleflex Inc.) in which a carbon tube (length: $280 \mathrm{~mm}$, exterior diameter: $8 \mathrm{~mm}$, interior diameter: $7 \mathrm{~mm}$ ) was inserted, to harden the endotracheal tube and correct its curvature. A piece of plexiglass tubing was shaped to fix and hold straight the diffusing part of the optical fiber, inside the inflated cuff, to guarantee homogenous illumination. Light was delivered using a 40 mm-tip-long cylindrical light diffuser (Model RD, Medlight ${ }^{\circledR}$ S.A., Switzerland). The cuff of the endotracheal was filled with $21 \mathrm{ml}$ of intralipid solution at the concentration of $0.01 \%(3)$. Figure 1 represents the light wand assembled.

\subsection{Illumination profile characterization}

In order to characterize the illumination profile of the wand, it was set up in a specific container. A plastic tank of black color limiting light reflection was filled with approximately $2300 \mathrm{ml}$ of dilute intralipid solution $0.01 \%$, to reproduce the illumination of the pleural cavity. The tip of the 
light wand was fixed horizontally in the tank through a cable gland and the optical fiber was connected to a medical diode laser $635 \pm 3 \mathrm{~nm}$ (Ceralas ${ }^{\circledR}$, Biolitec).

Light distribution around the diffusing tip of the light wand was then characterized by two complementary methods: direct measurements of the applied light power using sensors and estimation of the light gradient dose using a digital photography. The first allows a precise but partial sampling of the measurements while the second permits spatially characterization of light distribution.

\subsubsection{Fluence rate measurments}

An isotropic probe (Model IP 85, Medlight ${ }^{\circledR}$ S.A., Switzerland), collecting light in a large solid angle, was placed in a plastic tube and connected to a hand-held optical power meter (Model 841PE, Newport ${ }^{\circledR}$ Corporation, Irvine, CA, USA). The tube is used as a support to prevent any movement from the probe, and so for a better accuracy and reproducibility of the measurements. The sensor tip is located outside the tube and in direct contact with the liquid. The isotropic probe was fixed vertically and moved above the diffusing tip of the light wand, using a benchmark of millimetric precision with a vertical and horizontal degree of freedom (figure 2 (a)). Twodimensional measurements (nW) of light power were taken every $5 \mathrm{~mm}$ around the cuff of the light wand, as represented on figure 2 (b) and 2 (c), for a total of 124 measurement points. This dosimetry method had the advantage of giving precise measurements of the light emitted. The measurements were done with a laser power output of $1 \mathrm{~W}$ and an angle of $0^{\circ}$ along the endotracheal tube axis.

To test the reproducibility of this dosimetry method, the measurements were repeated three times, under the same experimental condition as described above, named "standard condition". Experiments were also performed rotating the endotracheal tube at an angle of $0^{\circ}, 90^{\circ}, 180^{\circ}$ and $270^{\circ}$ along the endotracheal tube axis. 
Last experiments were done by changing the laser power. In addition to $1 \mathrm{~W}$, the following powers were considered: $0.5 \mathrm{~W}, 1.5 \mathrm{~W}, 2 \mathrm{~W}, 2.5 \mathrm{~W}$ and $3 \mathrm{~W}$.

As the aim is to obtain light fluence rate measurements, the power values had to be converted into fluence rate values. A calibration factor was estimated using the following protocol: a flat sensor with $1 \mathrm{~cm}^{2}$ detection area (Model PD300, OPHIR, Israel) was used. Values recorded with this device were assumed to be the closest value for the local fluence rate $\left(\mathrm{W} / \mathrm{cm}^{2}\right)$.

For the same position and using a defined power value output from the laser, a measure was acquired using the flat sensor and another using the isotropic probe.

Different measurements were done by changing, either the positions inside the tank or the power applied by the laser.

The complete pairwise values were used in a correlation analysis to estimate the calibration factor (CalibFactor $\left.\left(\mathrm{cm}^{-2}\right)\right)$.

\subsubsection{Fluence rates distribution characterization}

The purpose is to obtain a complete description of the light distribution around the applicator tip. A digital camera (SPIRICON, USA) with a sensor appropriate for the laser wavelength. Installed above the tank containing the applicator. The photography was taken with a camera position pertaining to have the same field of view as on Figure 2-b. This field of view corresponds to a distance of $19 \mathrm{~cm}$ above the intralipid solution surface.

A Photography was acquired for each power value $(0.5 \mathrm{~W}, 1.0 \mathrm{~W}, 1.5 \mathrm{~W}, 2 \mathrm{~W}, 2.5 \mathrm{~W}$ and $3 \mathrm{~W})$.

The image as it is, is just a relative representation of the light attenuation around the applicator tip, calibrations are needed to make the image meaningful. Thus, it was processed by a homemade software (Optical Profiler) in the following way. First, using a ruler placed in the picture, a spatial calibration was done to estimate the pixel size, then the color map image was converted into a gray scale image and a normalization was done by dividing all the intensity values of the 
image by the maximum value. This value corresponds to the fiber trace represented by the brightest straight line on the image.

The second calibration is a pixel values calibration. It is based on the spatial matching of the punctual measurements done using the isoprobe with the picture. To this end, for each power values used as output from the laser, the points measurement array of figure 2-b is positioned on the corresponding picture to define values iso-surfaces.

This matching allowed defining for each gray level a corresponding power value (i.e. a fluence rate value after conversion using the calibration factor).

\section{RESULTS}

\subsection{Fluence rate measurement}

The measurements of the light power around the applicator, made with the isotropic probes, showed ellipsoidal surfaces as on Figure 3. The light power decreased exponentially in a radial direction from the center of the diffuser.

The light power decreases exponentially from the center of the diffusing part of the optical fiber. Thus, for each power, an exponential fit was done to estimate the light attenuation coefficient (effective attenuation factor $\mu_{\text {eff }}$. This modeling allowed describing the light attenuation around the applicator as:

$$
P=P_{0} \cdot e^{-\mu_{e f f} \text { Distance }}
$$

Where $\mathrm{P}_{0}$ is the measured power at distance 0 corresponding to the fiber.

When repeating three times the measurements of light power under "standard condition", the mean variation was of $9 \%$, while when rotating the light wand about its longitudinal axis to an angle of $0^{\circ}, 90^{\circ}, 180^{\circ}$ and $270^{\circ}$, the mean variation was of $6 \%$. This last result allows us to extrapolate the 2D measurements above the tip of the light wand (Figure 2-b) to a 3D 
representation of the profile, since rotating the light wand does not modify the power measurements.

Table 1 summarizes the obtained values for the different considered laser power values. From this table a mean attenuation factor was considered for the all values $\mu_{\mathrm{eff}}=0.70 \mathrm{~cm}^{-1}$.

The pairwise comparison allowed defining a calibration factor $\left(\right.$ CalibFactor $\left.=14446 \mathrm{~cm}^{-2}\right)$. The Multiplication of the values from equation 1 by this factor converted the power measurements into a fluence rate measurements.

\subsection{Fluence rates distribution characterization}

Figure 4 shows the picture taken above the light wand and its spatial matching with the measurements done using the isotropic probes.

Figure 5 depicts the results of the image processing and the calibration of the gray levels with the power (fluence rate) measurements for maximum power used $3 \mathrm{~W}$. 


\section{DISCUSSION}

Regarding all clinical applications of PDT, light delivery is crucial to the success of the therapy, and many teams have designed light devices adapted to various target organs, and specified their illumination patterns in terms of irradiance (4) or uniformity (5, 6). In 1991, DeLaney et al. published a study on a light device conceived for intra-pleural and intra-peritoneal PDT (4), aware of the necessity to deliver homogenous light to these complex surfaces. This light wand made of an endotracheal tube has been the light device used for intrapleural PDT for decades and is still used today.

Here, we have proposed a procedure to determine the illumination profile of a light device for intra-pleural PDT using two dosimetry methods, one for measuring the light power and the other one for characterizing the light distribution. The approach allowed estimating the delivered power at a specific location with a precision of more than $94 \%$ (mean error of $5.32 \%$ between experimental measured values and analytically estimated values).

Regarding the two employed techniques, they are complementary since the first, using the isotropic probe, allows accurate and exhaustive sampling of the measurements while the second, although giving only an image of the light distribution on the surface on the intralipid solution instead of on the surface of the light device, gives a large field of view. This last technique was initially investigated by McKenzie and O'Byrne in their study (7). The authors demonstrated, using a mathematical formalism, that fluence rate isodose distribution delivered by an interstitial bare-fiber can be determined by photographing the light diffusion out of the tissues without distortions.

From the ponctual measurements, we were able to define an effective attenuation coefficient specific to the light device's environment, that is $0.01 \%$ intralipid solution, and measurements from the illumination profile successfully corresponded with experimental isotropic probe 
measurements. The characterization of our illumination profile does not only provide information on the spatial distribution of the light from the diffuser in dilute intralipid, but also on the percentage of light power delivered from the input laser power at a given distance. Therefore, one can predict the received irradiance at each point of the surface of the target to treat, here the pleural surface. This kind of approaches allows to obtain a unitary action model that could be cumulated in time and space to have a complete estimation of the applied light dose.

The method described herein was applied specifically for the light wand for mesothelioma PDT, but it can easily be extended to other light devices based on cylindrical diffusers for other clinical applications (peritoneal cavity, endoluminal PDT).

The main limitation of this approach is that the illumination profile does not take into account extrinsic factor that may alter the light distribution and therefore light measurements. In the case of MPM, hemoglobin appears in the intralipid solution during light delivery, due to microbleedings after the tumor resection. However, it must be stressed that during intrapleural PDT procedures, intralipid solution inside the thoracic cavity is suctioned and replaced as soon as it turns red.

Regarding light dosimetry for MPM, the current technique developed by the expert team of University of Pennsylvania is composed of 7 isotropic probes attached to strategic locations in the thorax, and connected to a dosimetry system (8). The light is collected at the millimetric tip of this probe at each of these locations. The light dose is cumulated and displayed on a computer screen, in real time during illumination. The main limitation of this technique is that it only gives information about the light delivered at these 7 locations, but not to the rest of the pleural surface (at least $800 \mathrm{~cm}^{2}$ ). As a future application, we plan to track the position of light wand inside the pleural cavity, using a spatial locator, and include into the system our illumination profile, to palliate the lack of spatial dosimetry for intrapleural PDT. 


\section{CONCLUSION}

The proposed light dosimetry method allows presenting an accurate theoretical illumination profile of a light device used for the procedure of PDT. In the future this model could be combined with a spatial tracking system, so that a real time light dosimetry monitoring could be performed, so that the operator can adapt the movements of the light wand inside the cavity to obtain homogeneous light delivery.

\section{Acknowledgments}

This work has been supported by "Société de Pathologie Thoracique du Nord" and the "Fondation ARC pour la recherche sur le cancer" and the authors wish to thank Romain Delos and Mélanie Azaiis for the help they provided regarding the light dosimetry measurements. 


\section{REFERENCES}

1. Friedberg JS, Culligan MJ, Mick R, Stevenson J, Hahn SM, Sterman D, et al. Radical Pleurectomy and Intraoperative Photodynamic Therapy for Malignant Pleural Mesothelioma. Ann Thorac Surg. 2012 May;93(5):1658-67.

2. Friedberg JS, Mick R, Culligan M, Stevenson J, Fernandes A, Smith D, et al. Photodynamic Therapy and the Evolution of a Lung-Sparing Surgical Treatment for Mesothelioma. Ann Thorac Surg. 2011 Jun;91(6):1738-45.

3. Friedberg JS. Photodynamic Therapy as an Innovative Treatment for Malignant Pleural Mesothelioma. Semin Thorac Cardiovasc Surg. 2009 Jun;21(2):177-87.

4. DeLaney TF, Smith PD, Thomas GF, Tochnen ZA, Sindelar WF, Pass HI, et al. A lightdiffusing device for intraoperative photodynamic therapy in the peritoneal or pleural cavity. $J$ Clin Laser Med Surg. 1991;9(5):361-6.

5. Canavesi C, Cassarly WJ, Foster TH, Rolland JP. Lightpipe device for delivery of uniform illumination for photodynamic therapy of the oral cavity. Appl Opt. 2011;50(16):2322-5.

6. Allrdice MJT, Abulafi AM, Dean R, Rowland AC, Williams NS. Light delivery systems for adjunctive intraoperative photodynamic therapy. Lasers Med Sci. 1993;8(1):1-14.

7. McKenzie AL1, Byrne PO. Can photography be used to measure isodose distributions of space irradiance for laser photodynamic therapy? Phys Med Biol. 1988 Jan;33(1):113-31.

8. Zhu TC. Dosimetry in pleural photodynamic therapy. J Natl Compr Canc Netw. 2012;10(Suppl 2):S - 60 . 


\section{FIGURE LEGENDS}

Figure 1: The optimized light wand designed within laboratory. The cuff is filled with dilute intralipid liquid.

Figure 2: (a) Setting of the light wand; (b) Measuring points around the light wand with the isotropic probe under "standard condition"- Longitudinal view - (c) Frontal view.

Figure 3: Example of light power measurements with the isotropic probe around the light applicator for a output power of $2 \mathrm{~W}$. The results correspond to the measurements as done in figure 3.b.

Figure 4: (a) Digital photography taken above the light wand in dilute intra-lipid; (b) Spatial matching of the power measurements with the picture.

Figure 5: Illumination profile of the light wand for power output of $3 \mathrm{~W}$. Fluence rates iso-surfaces localizations regarding the cuff of the applicator. 


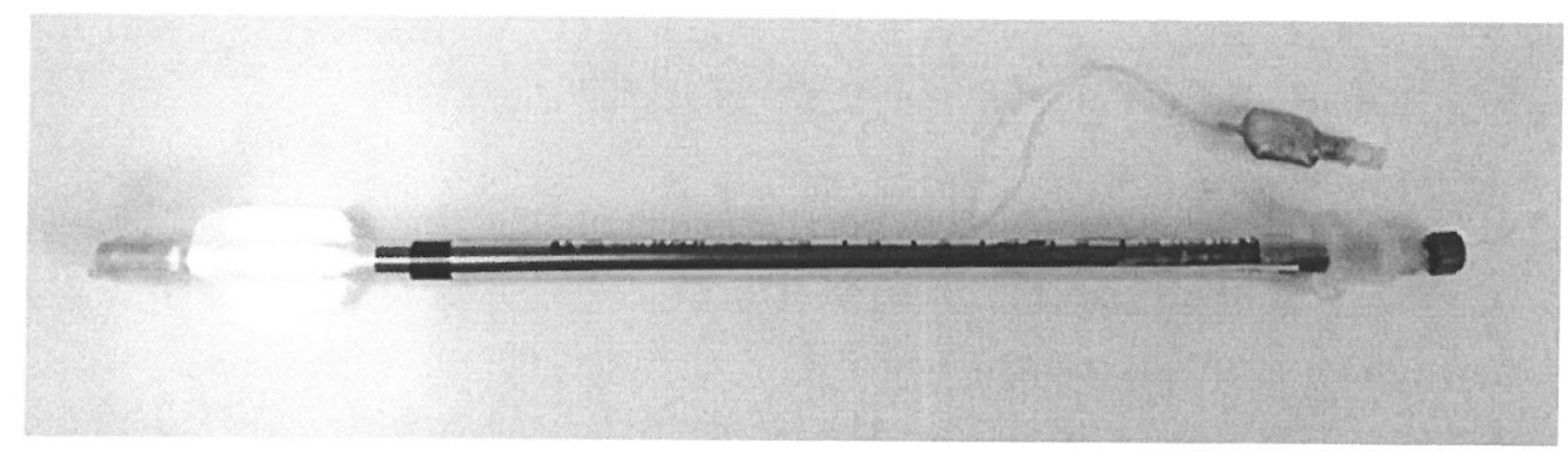

(a)

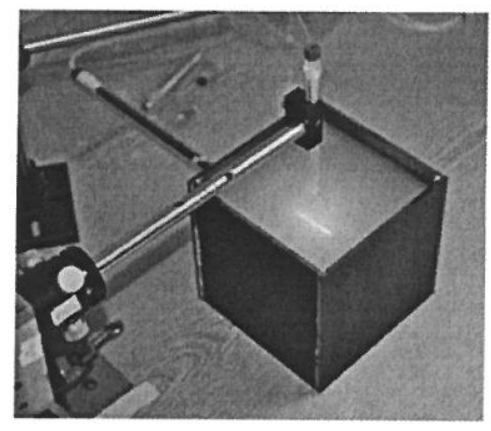

(b)

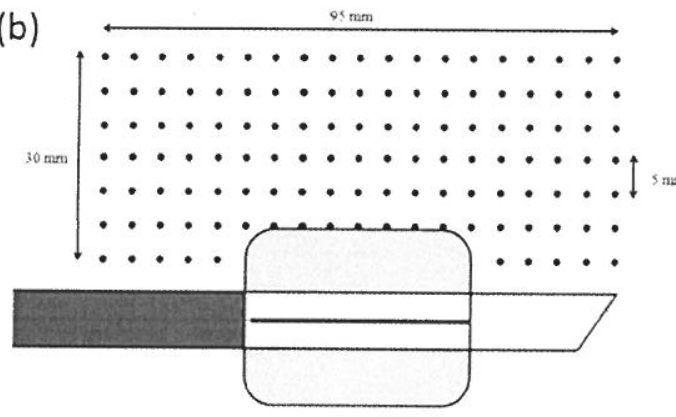

(c)

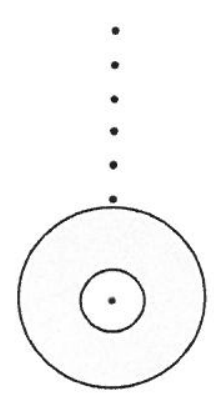

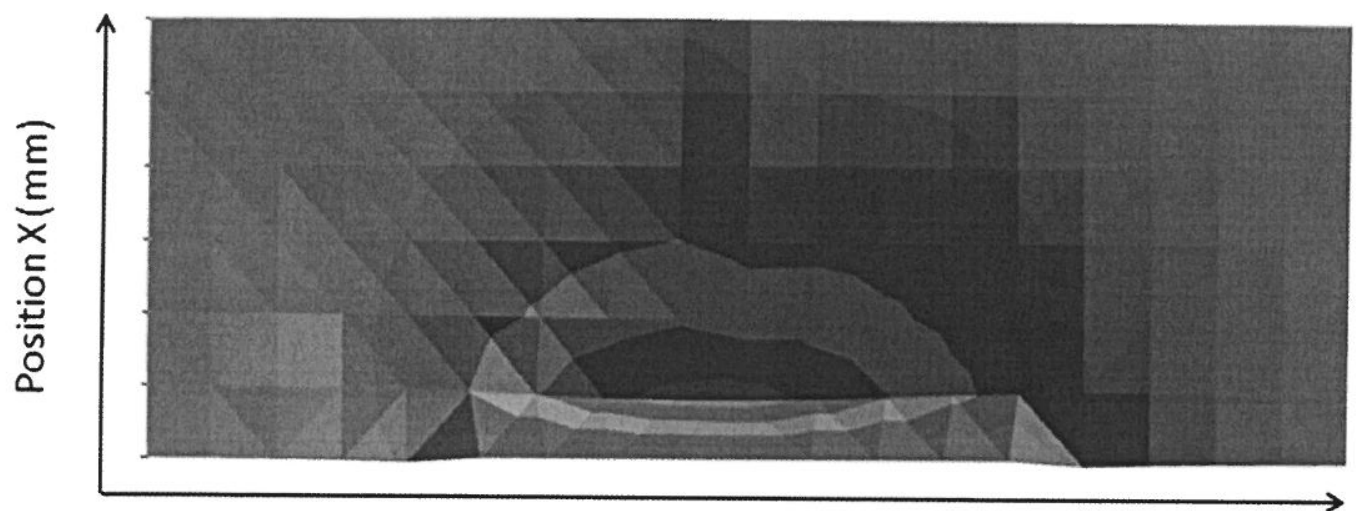

Position $Y(\mathrm{~mm})$

Powervalues $(\mathrm{nW})=200-250=150-200=100-150 \square 50-100$ a-50 
(a)

(b)

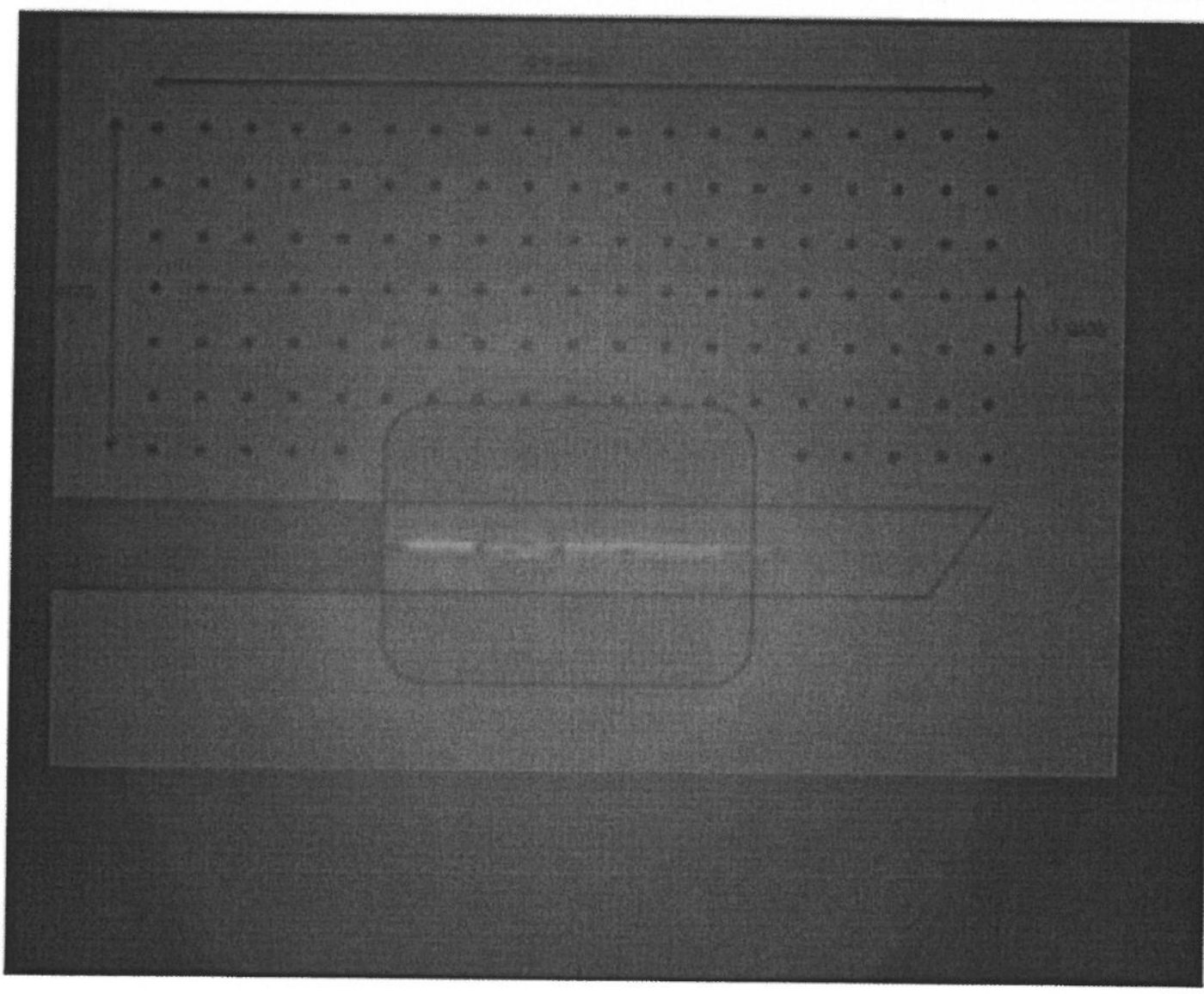




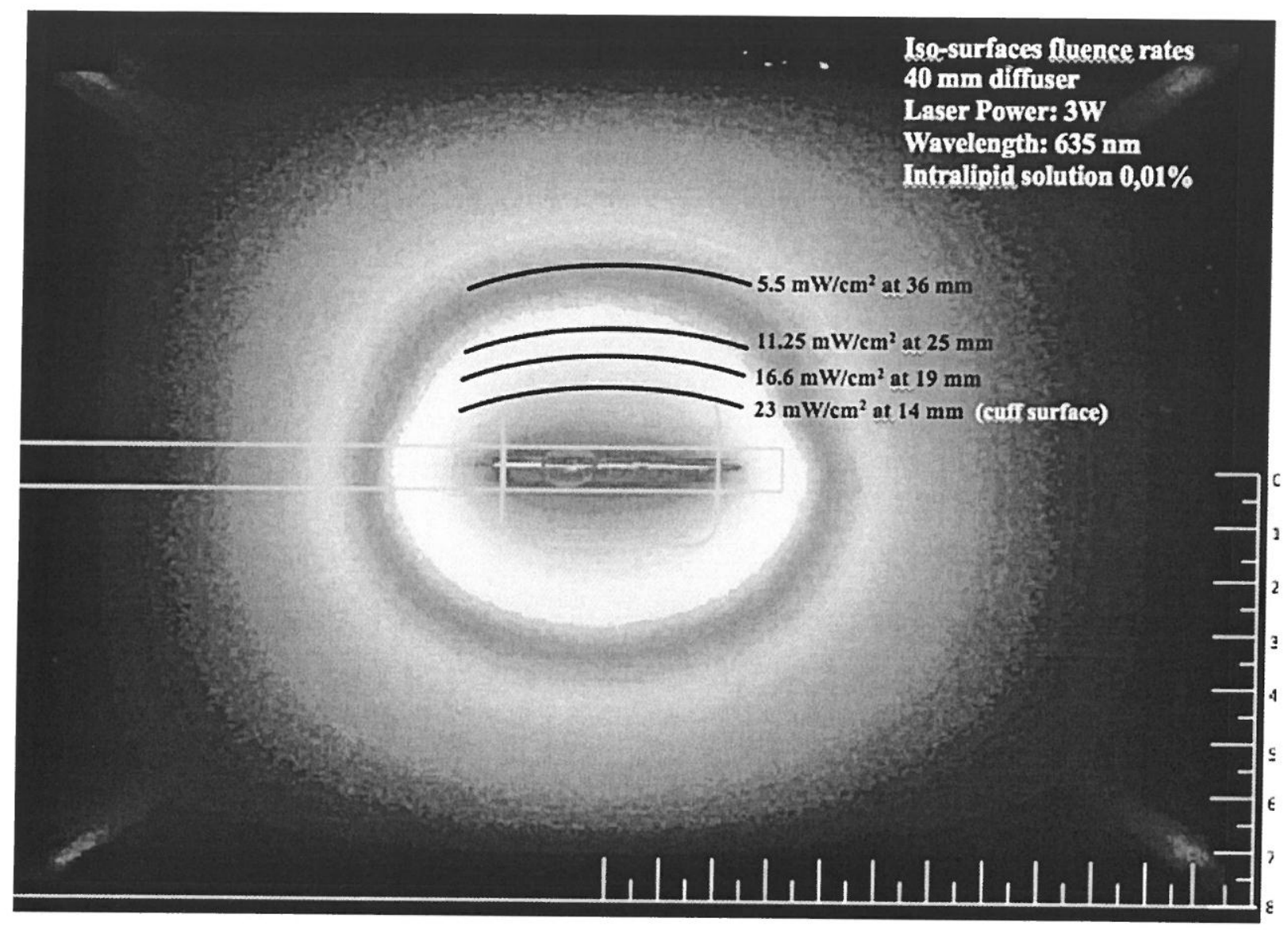

\begin{tabular}{cc}
\hline $\begin{array}{c}\text { Laser power output } \\
(\text { Watt })\end{array}$ & $\begin{array}{c}\text { Effective attenuation } \\
\text { coefficient }\left(\mu_{\mathrm{eff}}\right)\left(\mathbf{c m}^{-1}\right)\end{array}$ \\
\hline 0.5 & 0.648 \\
1.0 & 0.655 \\
1.5 & 0.654 \\
2.0 & 0.66 \\
2.5 & 0.652 \\
3.0 & 0.649 \\
\hline
\end{tabular}

Table 1: Effective attenuation coefficient estimated from the isotropic probes measurement for different laser output power. 